\title{
Raman Amplification And Pulsed Lasing In Cladding-Pumped Germanosilicate Fiber
}

\author{
J.N.Jang, J.K.Sahu, R.Selvas, J.Nilsson, D.C.Hanna \\ Optoelectronics Research Centre
}

\author{
A. B. Grudinin \\ Southampton Photonics, Phi House, Enterprise Road, Chilworth Science Park, \\ Southampton SO16 7NS, UK
}

\section{Introduction:}

Cladding-pumped rare-earth-doped fibers and Raman fiber devices have been two of the most compelling advances in the area of fiber amplifiers and lasers in recent years. Both cladding-pumped fibers and Raman fiber devices are primarily high-power devices. Cladding-pumping enables pumping with multi-mode pump sources that deliver high-power at a low cost. The core of the double-clad fiber can still be single-moded, so that robust single-mode amplification or lasing can be realized. Claddingpumped silica fiber lasers and amplifiers have been demonstrated at $980 \mathrm{~nm}[1,2,3]$ and $1020-1150$ $\mathrm{nm}$ [4] with Yb-doping, 1050 - $1120 \mathrm{~nm}$ with Nd-doping [5], 1530 - $1620 \mathrm{~nm}$ with Er-doping (normally with, but also without, Yb co-doping) [6], and $2000-2200 \mathrm{~nm}$ with Tm-doping [7]. These rare earths can be pumped by widely available high-power diode sources at around $800 \mathrm{~nm}$ or $910-$ $980 \mathrm{~nm}$.

Raman gain requires high pump powers. Raman fiber lasers are therefore only efficient at high power levels. On the other hand, Raman devices are very flexible in that gain is available at arbitrary wavelengths with the right pump source. Thus, a popular configuration for distributed Raman amplification at $1550 \mathrm{~nm}$ is to use a cascaded Raman laser as a pump source at $1450 \mathrm{~nm}$ (where rareearth doped lasers are not available), which is in turn pumped by a high-power cladding-pumped $\mathrm{Yb}$ doped fiber laser at $1060 \mathrm{~nm}$. Besides this example, cladding-pumped fiber devices have many other real and potential applications in telecom and other areas, such as high-power fiber amplifiers, and sources for remote sensing (e.g., lidar). Raman amplification can be used for distributed or lumped amplification in telecom systems, but also more generally as a means for wavelength conversion (notably in cascaded Raman lasers).

Cladding-pumped Raman fiber devices, an exciting combination of multi-mode pumping and Raman amplification, have recently been proposed [8]. With such devices, single-mode gain can be created at arbitrary wavelengths with multi-mode pump sources (at the right wavelength). Conversely, any multimode source with sufficiently high power can be Raman-converted to a single-mode beam in a double-clad fiber configured as an amplifier or as a laser. One is no longer restricted to the absorption and emission bands of rare earths. While cladding-pumped Raman fiber devices require pump powers of, say, $100 \mathrm{~W}$, such powers are available from fiber sources as well as traditional bulk sources. For

Fig. 1. Experimental setup for cladding pumped Raman fiber amplifier.

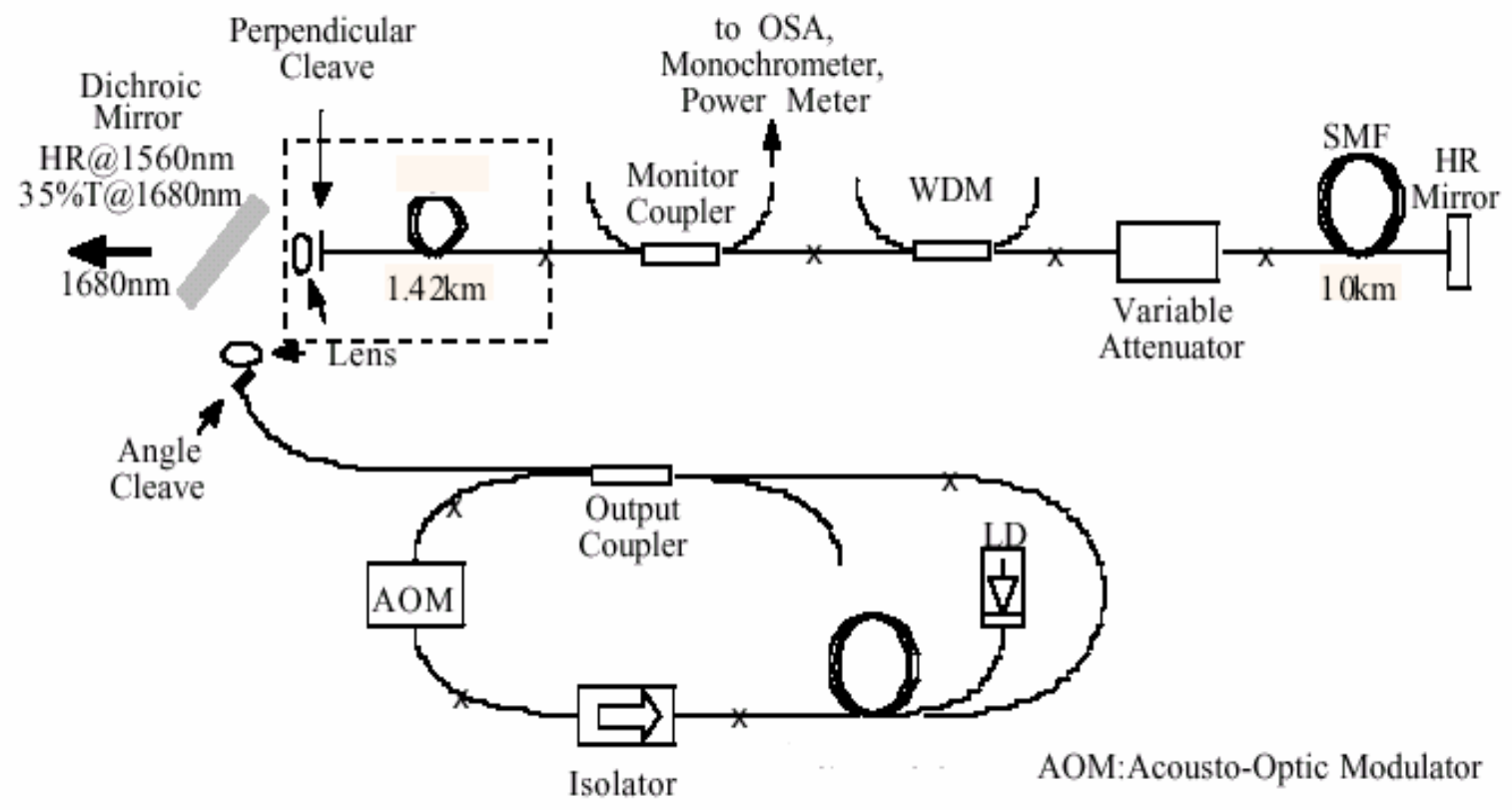




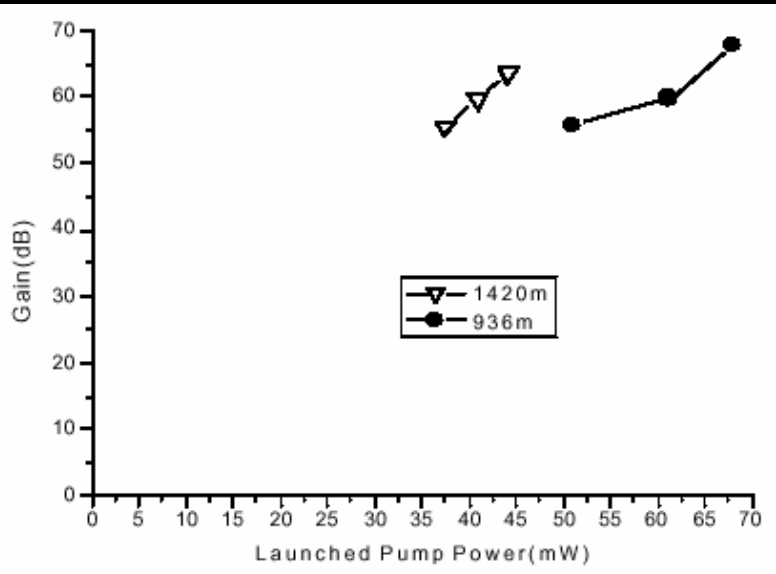

Fig. 2: Gain vs. pump power.

example, a multimode Nd-doped fiber laser with $1 \mathrm{~kW}$ of output power was recently reported [9]. With pulsed sources, the required peak powers can be achieved with relatively modest average powers.

Here, we report for the first time Raman amplification in a cladding-pumped fiber, without any rare-earth doping. We believe that this is an important step towards a whole class of new optical amplifier devices based on brightness-enhancing nonlinear conversion of multimode pumps, including not only Raman converters but also Brillouin and optical parametric converters [10, 11].

\section{Set-up:}

Our experimental set-up is shown in Fig. 1. We used a Q-switched Er-Yb co-doped fiber laser in a multi-fiber (GT-wave) arrangement [12]. See inset in Fig. 1. It was pumped by a pig-tailed multi-mode laser diode with $2.4 \mathrm{~W}$ of fiber-coupled output power at $910 \mathrm{~nm}$. It generated up to $320 \mathrm{~mW}$ of average output power at high repetition rates (e.g., $70 \mathrm{kHz}$ ). In our experiment, we used the laser at low repetition rate, where it generated pulses with energies up to $40 \mu \mathrm{J}$ with pulse durations down to $200 \mathrm{~ns}$ and with a time jitter of $\sim 5 \mathrm{~ns}$. The lasing wavelength was $1565-1570 \mathrm{~nm}$. The output from the fiber laser was free-space coupled into the double-clad Raman fiber (DCRF) via a dichroic mirror. Though the Q-switched fiber laser produced a single-moded output, we took great care to ensure that the pump beam was launched into the inner cladding, rather than the core, of the DCRF. Following all measurements presented here, we cut the DCRF a small distance from the pump launch end, and evaluated total launched pump power as well as pump power launched into the core by splicing the DCRF to a piece of standard single-mode fiber. We could launch up to $88 \%$ of the output power from the Q-switched fiber laser into DCRF. Of this power, typically $10-15 \%$ was in the core. We varied the launched pump power, without changing the pulse shape, repetition rate, or fraction of power in

the core of the DCRF by inducing a bend-loss on the output fiber of the Q-switched fiber laser.

In the DCRF, the pump source generates Raman gain with a peak wavelength of $1680-1690 \mathrm{~nm}$. Unfortunately, we did not have a signal seed source at that wavelength, and we therefore had to resort to the rather complicated set-up of Fig. 1, which effectively generates its own signal seed. However, we emphasize that the Raman converter itself is very simple, consisting only of the DCRF. The pump-tosignal conversion takes place in a single amplification pass. Thus, we consider the boxed portion of the set-up to be a cladding-pumped Raman fiber amplifier with input and output ends according to the figure. Besides being simple, the DCRF is also quite versatile in that it can be used with any pump source emitting within the wide transparency window of the fiber. Alternatively, the set-up in Fig. 1 can be viewed as a synchronously pumped pulsed Raman laser.

The DCRF had a pure silica outer cladding and germanosilicate inner cladding and core, with different germanium contents. The inner cladding had a diameter of $21.6 \mu \mathrm{m}$ and an NA of 0.22 with respect to the outer cladding. The core had a diameter of $9 \mu \mathrm{m}$ and an NA of 0.14 with respect to the inner cladding, leading to an estimated cut-off wavelength of $1630 \mathrm{~nm}$. The core propagation loss was $3.1 \mathrm{~dB} / \mathrm{km}$ at $1550 \mathrm{~nm}$. The loss for light in the inner cladding was $2.3 \mathrm{~dB} / \mathrm{km}$ at $1550 \mathrm{~nm}$. The fiber was $1.4 \mathrm{~km}$ long. Since Raman gain is essentially instantaneous and since the pump pulse is much shorter than the DCRF, the pump pulse creates Raman gain that travels with it through the fiber. Therefore, the Raman gain is much higher for signal light traveling with the pump than it is in the counter-propagating direction. Consequently, the signal at the output end of the DCRF will also be pulsed, and temporally coincident with the pump pulse. 
The signal pulse emitted from the DCRF continues on through the other components in the cavity. At its output end, the DCRF is spliced to a fused fiber coupler fabricated with standard single-mode fiber (NA 0.12, core diameter $8 \mu \mathrm{m}$ ). The splice loss between standard single-mode fiber (SSMF) and the DCRF was $\sim 0.5 \mathrm{~dB}$ for the core mode. By contrast, since the cladding SSMF does not guide light, all pump light except the small fraction in the core is lost here. The coupler had a nearly flat wavelength response, and coupled out $40 \%$ of the incident power at $\sim 1680 \mathrm{~nm}$. The loss for the through-path was $4.3 \mathrm{~dB}$ at $1690 \mathrm{~nm}$. This monitor coupler was then spliced to another, wavelengthselective, coupler. It had a low transmission loss $(\sim 2 \mathrm{~dB})$ at the first stokes wavelength $(\sim 1680 \mathrm{~nm})$ but a high loss at the second stokes wavelength as well as at the pump wavelength. Thus, it served as a filter that suppressed higher-order stokes generation. The couplers were followed by a variable optical attenuator (minimum insertion loss at $1550 \mathrm{~nm} 1.4 \mathrm{~dB}$ ) that allowed us to change the cavity loss. Finally, there was $\sim 10 \mathrm{~km}$ of standard single-mode fiber (loss at $1690 \mathrm{~nm} \mathrm{5dB}$ ). A high-reflecting mirror was butted to the fiber in the far end. We estimate the reflection loss to $2 \mathrm{~dB}$.

Fig. 3: Output power vs. pump power.

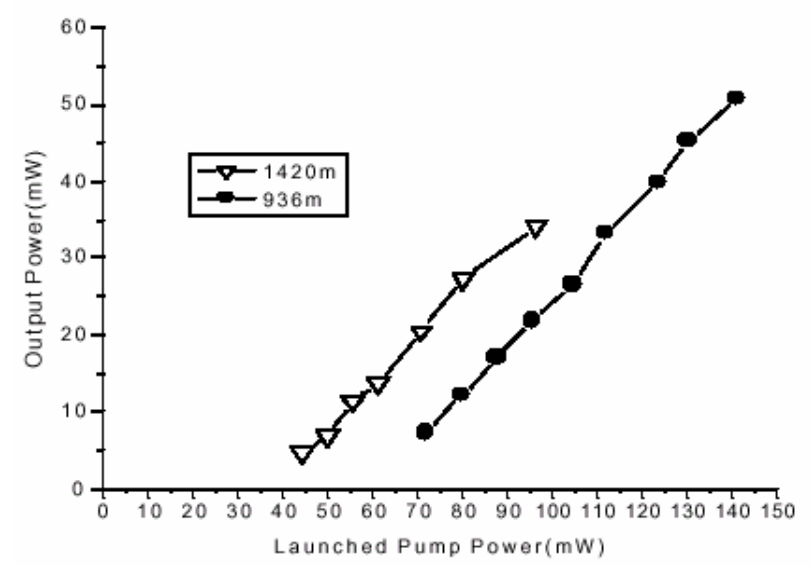

The signal pulse is reflected back through the cavity all the way to the pump launch end of the DCRF. There, $4 \%$ is reflected again from the perpendicularly cleaved fiber end. At the same time, a new pump pulse is launched into the DCRF. The reflected signal light acts as a seed for the conversion in the cladding-pumped Raman fiber amplifier. Because the mode-selection that occurs in the SSMF and the low mode-coupling at splice and reflection points, the reflected signal is almost exclusively coupled to the core mode. The roundtrip cavity loss was $\sim 55 \mathrm{~dB}$. The roundtrip time was $115.27 \mu$ s, so the Q-switched fiber laser had to be carefully adjusted to a repetition rate of $8.6754 \mathrm{kHz}$ for synchronous pumping. We used this repetition rate throughout. The pulse energy became $30 \mu \mathrm{J}$ and the pulse duration was $210 \mathrm{~ns}$. Thus, the maximum peak power became $140 \mathrm{~W}$, or 540 times the average power. This ratio remained constant also with an attenuated pump beam.

In an alternative configuration, a section of the DCRF was moved from its original location to a position between the VOA and the $10 \mathrm{~km}$ SSMF. At this point, the DCRF is un-pumped, so this way we could change the effective length of the DCRF without changing the total length or loss of the cavity.

Unfortunately, this set-up does not allow us to monitor the pump power remaining at the end of the DCRF. We cannot measure the pump power without breaking the DCRF. But if we do that, the cavity is destroyed and there is no longer any input signal seed. Nevertheless, we did measure transmitted pump power with the cavity opened at the end of the DCRF $(1.4 \mathrm{~km})$, and found it to be $\sim 68 \mathrm{~mW}$ for $100 \mathrm{~mW}$ launched. 

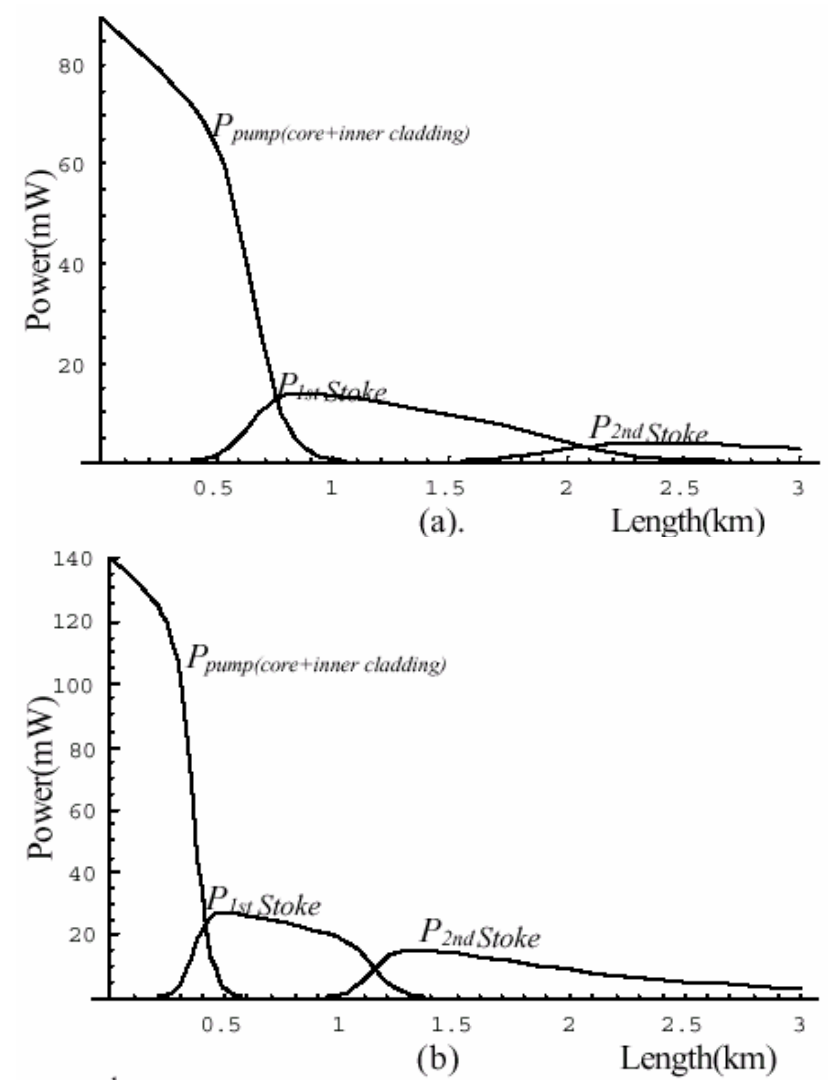

Fig. 4. Simulation result of pump, $1^{\text {st }}$ and $2^{\text {nd }}$ Raman power evolution along the fiber (a); $90 \mathrm{~mW}$ pump power, (b);140 $\mathrm{mW}$ pump power

\section{Results:}

Figure 2 shows the DCRF gain vs. pump power for 1420 and $940 \mathrm{~m}$ long DCRFs. The gain was determined by varying the total cavity loss via the VOA, and adjusting the pump power until threshold for lasing was reached. The gain slopes are $1.5 \mathrm{~dB} / \mathrm{mW}$ and $1.0 \mathrm{~dB} / \mathrm{mW}$, respectively. The effective lengths become $1000 \mathrm{~m}$ and $740 \mathrm{~m}$. These numbers are in good agreement with theory, given the uncertainty in evolution of polarization and modal power distribution, and that the high Ge-content increases the Raman cross-section. Beyond the plotted range, laser threshold could not be reached because of the onset of strong ASE. Still, because of the high pump power, a gain of almost $70 \mathrm{~dB}$ could be reached. Figure 3 shows the output power from the DCRF vs. pump power for 1420 and 940 $\mathrm{m}$ long DCRFs. For these measurements, the VOA was set to its minimum loss value. The output power was evaluated by measuring the power exiting the coupler monitor port with a thermal power meter and recalculating it to the power coming out from the DCRF. The thresholds are $37 \mathrm{~mW}$ and 61 $\mathrm{mW}$, and the slope efficiencies are $60 \%$ and $64 \%$ for the longer and shorter fiber, respectively. For both fiber lengths, the power in the core at the output end becomes significantly higher than in the input end: The highest pump power launched into the core was $10-20 \mathrm{~mW}$. This demonstrates brightness enhancement via Raman amplification of a signal in the core with a pump beam substantially launched into the inner cladding.

At high pump power, higher-order Raman generation can occur. In Fig. 3, we restricted the pump power to values for which this was negligible. Thus, since higher-order generation occurs more readily in longer fibers, the maximum pump power for the $1420 \mathrm{~m}$ fiber is lower than that of the $940 \mathrm{~m}$ fiber.

To study this further, we simulated our device in the cw regime, using simple, well-known equations for stimulated Raman scattering [13]. Since higher-order Raman conversion is very important in limiting the efficiency of these devices, this was also included in our simulations. The interactions of the pump and $1^{\text {st }}$ and $2^{\text {nd }}$ Stokes waves are given by the following equations:

$$
\frac{d P_{P}}{d z}=-\alpha_{P} p_{P}-\frac{\lambda_{P}}{\lambda_{S 1}} \frac{g_{1}}{A_{e f f . S 1}} P_{P} P_{S 1}-\frac{\lambda_{P}}{\lambda_{S 2}} \frac{g_{2}}{A_{e f f . S 2}} P_{P} P_{S 2}
$$




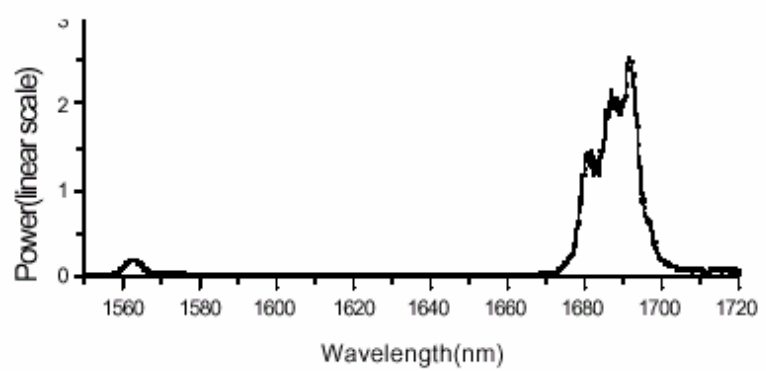

Fig. 5: Output spectrum after $940 \mathrm{~m}$ of DCRF.

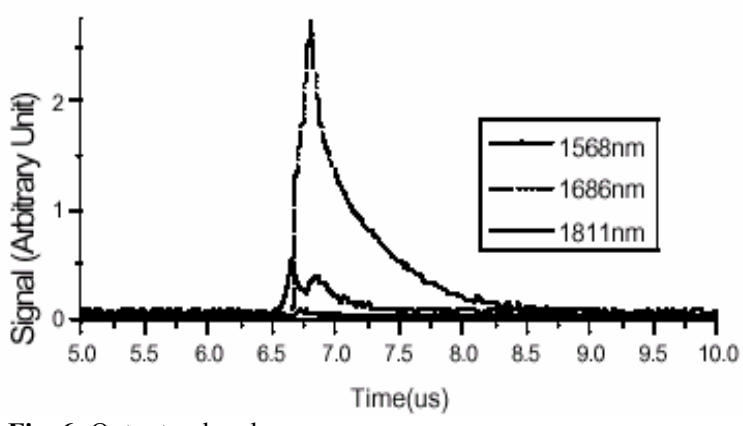

Fig. 6: Output pulse shapes

$$
\begin{aligned}
& \frac{d P_{S 1}}{d z}=-\alpha_{S 1} p_{S 1}+\frac{g_{1}}{A_{\text {eff. } P}} P_{P} P_{S 1}-\frac{\lambda_{S 1}}{\lambda_{S 2}} \frac{g_{1}}{A_{\text {eff. } S 2}} P_{S 1} P_{S 2} \\
& \frac{d P_{S 2}}{d z}=-\alpha_{S 2} p_{S 2}+\frac{g_{1}}{A_{\text {eff. } S 1}} P_{S 1} P_{S 2}+\frac{g_{2}}{A_{\text {eff. } P}} P_{P} P_{S 2}
\end{aligned}
$$

where $P_{p}, P_{S 1}$ and $P_{S 2}$ are pump, $1^{\text {st }}$ Stokes, and $2^{\text {nd }}$ Stokes powers. $\alpha$ and $\lambda$ are corresponding absorption coefficients and wavelengths. $g_{l}$ and $g_{2}$ are Raman gain coefficients for $12 \mathrm{THz}$ and $26 \mathrm{THz}$ frequency shift, respectively, corresponding to the experimentally obtained shifts. Pump wavelength is $1564 \mathrm{~nm}$ and $1^{\text {st }}, 2^{\text {nd }}$ Stokes wavelengths are $1690 \mathrm{~nm}$ and $1810 \mathrm{~nm}$. $A_{\text {eff }}$ is the effective area of the fiber mode. The $3^{\text {rd }}$ term in Eq. 1. represents the interaction between the pump and $2^{\text {nd }}$ Stokes directly. We consider only $1^{\text {st }}$ and $2^{\text {nd }}$ Raman shifts because the $3^{\text {rd }}$ Raman is very small and can be neglected. Signal seed source powers from the synchronously pumping scheme were calculated from the measured power and the total cavity loss. $1^{\text {st }}$ Stokes powers are $2.3 \times 10^{-4} \mathrm{~mW}$ for $90 \mathrm{~mW}$ pumping power and $8.43 \times 10^{-4} \mathrm{~mW}$ for $140 \mathrm{~mW}$ pumping, respectively. We used the experimentally obtained Raman gain coefficient of $g_{l}=0.233 \mathrm{~Np} / \mathrm{km} / \mathrm{mW}$ for the Raman gain peak. For the Raman gain between the pump and $2^{\text {nd }}$ Stokes, we used the Raman gain spectra in Ref. 13. The absorption coefficients are $0.53 \mathrm{~km}^{-1}$ for $\alpha_{\mathrm{p}}, 0.714 \mathrm{~km}^{-1}$ for $\alpha_{\mathrm{S} 1}$, and $0.9 \mathrm{~km}^{-1}$ for $\alpha_{\mathrm{S} 2}$. We used the laser repetition rate of 8.6754 $\mathrm{kHz}$ for synchronous pumping and the pulse duration time was $210 \mathrm{~ns}$, which means the maximum peak power is 540 times higher than the average power.

Figure 4 shows simulation results of pump, $1^{\text {st }}$ and $2^{\text {nd }}$ Raman power evolution along the fiber for $90 \mathrm{~mW}$ and $140 \mathrm{~mW}$ pump power using the Eq. 1 to Eq. 3.

As we can see in Fig. 4(a), there is no $2^{\text {nd }}$ Raman conversion with $90 \mathrm{~mW}$ of pump power within $1.42 \mathrm{~km}$ of fiber, but with $140 \mathrm{~mW}$ of pump power in Fig. 4(b), higher-order Raman starts to appear after $\sim 1 \mathrm{~km}$ of fiber. At $1.4 \mathrm{~km}$, practically all $1^{\text {st }}$ order Raman power has been converted to $2^{\text {nd }}$ order Raman. These characteristics are in agreement with the experimentally observed behaviour.

Figure 5 shows experimentally obtained output spectrum, measured with an optical spectrum analyzer on the monitor output port for a fiber length of $940 \mathrm{~m}$ and a pump power of $140 \mathrm{~mW}$. Unfortunately, the spectrum analyzer was limited to wavelengths up to $1750 \mathrm{~nm}$, whereas the second stokes occurred at $\sim 1810 \mathrm{~nm}$. However, we also used a monochromator to resolve higher-order stokes radiation and for temporal measurements. Figure 6 shows output pulse shapes at the wavelengths of the pump and first and second stokes beams, measured with the monochromator and a germanium detector. We see that the signal at the second stokes wavelength is much weaker than that at the first stokes wavelength.

\section{Discussion:}

Compared to rare-earth doped fibers, the Raman gain efficiency is quite low, especially in large structures. It is still relatively straightforward to reach high gain with pulsed pump sources. Even $\mathrm{cw}$ pump lasers (fiber and non-fiber ones) of sufficiently high powers are becoming increasingly available. In a laser configuration, the cavity losses can be sufficiently low for thresholds of a few watts, given a gain efficiency of the order of $1 \mathrm{~dB} / \mathrm{W}$ (cw). Still, cladding-pumped Raman fibers only seem realistic with inner-cladding diameters of a few tens of microns, rather than with hundreds of microns as is common with rare-earth doped cladding-pumped fibers. This limits the scope for brightness enhancement and the high pump intensities required (pulsed or $\mathrm{cw}$ ) presently preclude direct diode pumping. On the other hand, when suitable high-power pump sources are available, there are several advantages of Raman devices over rare-earth ones. Besides the flexibility in wavelength, the low loss of germanosilicate fibers allows the use of much longer devices, so that the heating per unit length can be small. At higher powers, however, the Raman conversion can occur in fibers as short as a few 
meters. Then, the small quantum defect of Raman scattering in silica simplifies the thermal management.

The length of the Raman fiber converter is important. A longer fiber leads to a higher gain efficiency in the small-signal regime. Therefore, it will be easier to reach threshold with a longer fiber. However, for higher powers, secondary Raman scattering occurs, this too with lower threshold for longer fibers. If this is to be avoided, there will be an upper limit on the pump power for a given fiber length. A shorter fiber has a higher upper limit. In Fig. 3, for pump powers up to $100 \mathrm{~mW}$, the $1420 \mathrm{~m}$ fiber is better than the $940 \mathrm{~m}$ one. For higher pump powers, second-order Raman scattering occurs in the $1420 \mathrm{~m}$ fiber. Consequently, one can reach higher output powers with $940 \mathrm{~m}$ of fiber than with $1420 \mathrm{~m}$, if sufficiently high pump power is available. Though a wavelength-suppressing filter could be used to suppress higher-order Raman scattering, this may be difficult to realize in practice.

Besides higher order Raman scattering, Raman scattering in the cladding should also be avoided. Since the core and cladding compositions are quite similar, and since the pump intensity is essentially similar in the core and inner cladding, the Raman gain will be similar in the core and inner cladding. If a single-moded output is.required, one must then seed the core-mode (or provide mode-selective feedback in a laser).

The measured slope efficiency of up to $64 \%$ is as high as it reasonably can be with the loss in our fiber, given that all light quanta have to propagate through the whole length of fiber, as signal or pump photons. A lower-loss fiber or a shorter fiber (as appropriate for higher pump powers) would reduce total loss and thus improve slope efficiency. It is interesting to note that because of the nonlinear pump absorption, Raman devices can actually have quantum slope conversion efficiencies exceeding unity over a limited power range. Our fiber was indeed operating in that power range.

\section{Conclusions:}

In conclusion, we report for the first time results on amplification in a cladding- pumped Raman fiber. We used a Q-witched Er-Yb co-doped fiber laser operating at 1565 - $1570 \mathrm{~nm}$ as a pump source. The power conversion efficiency was up to $36 \%$, with a slope of $64 \%$. We believe that claddingpumped fiber devices present a very exciting alternative for amplification and brightness conversion at high powers.

\section{References}

[1] L. A. Zenteno, J. D. Minelly, A. Liu, J. G. Ellison, S. G. Crigler, D. T. Walton, D.V. Kuksenkov, M. J. Dejneka, "1 W singletransverse-mode Yb-doped double clad fiber laser at 978nm", Electron. Lett, Vol. 37, pp 819-820, 2001.

[2] L. B. Fu, R. Selvas, M. Ibsen, J.K. Sahu, S.A. Alam, J. Nilsson, D.J. Richardson, D.N. Payne, C. Codemard, S. Goncharov, I. Zalevsky, and A B. Grudinin, "An 8-channel fiber-DFB laser WDM transmitter pumped with a single 1.2W Yb-fiber laser operated at 977nm", Technical digest 28 th European Conference on Optical Communication ECOC-2002, Copenhagen, September, 2002. 08.3.5

[3] R. Selvas, J. K. Sahu, J. Nilsson, S. A. Alam, and A.B. Grudinin, "Q-Switched 980nm Yb-doped fiber laser", Technical digest Conference of Lasers and Electro-optics CLEO-2002, Long Beach, 2002, ThR6.

[4] J.K. Sahu, C.C. Renaud, K. Furusawa, R. Selvas, D. J. Richardson, and J. Nilsson, "Jacketed air-clad cladding pumped Ybdoped fiber laser with wide tuning range", Electronics Letter, Vol 37 (18), August 2001.

[5] R. Selvas, J. Nilsson, "Tuning characteristics of cladding-pumped Neodymium-doped fiber laser", Technical digest Conference on Laser Electro-optics CLEO- 2001, Baltimore, 2001. CWM4.

[6] S.U. Alam, J. Nilsson, P.W. Turner, W.A. Clarkson, A.B. Grudinin, "Erbium-ytterbium co-doped cladding-pumped fiber laser tunable from 1533 to $1600 \mathrm{~nm}$ with up to $6.7 \mathrm{~W}$ of output power, In proc. ASSL, Washington, 28-31, January 2001, TuA5.

[7] R Hayward, W. A. Clarkson, P.W. Turner, J. Nilsson, A. B. Grudini, D. C. Hanna, "High-power and tunable operation of a diode-bar-pumped double -clad Tm-doped silica fiber laser at 2 mm”, In proc. CLEO/Europe 2000, Nice 10-15, Sep 2000, CWA1

[8] R. R. Rice, "Multimode Raman fiber amplifier and method”, US patent no. 6,353,087 (2002)

[9] K. -I. Ueda, H. Sekiguchi, and H. Kan, "1 kW cw output from fiber embedded lasers", in Proc. Conference on Lasers and Electro-Optics, Long Beach, USA, 2002, post-deadline paper CPDC4

[10] B. C. Rodgers, T. H. Russell, and W. B. Rob, "Laser beam combining and cleanup by stimulated Brillouin scattering in a multimode optical fiber", Opt. Lett. 24, 1124-1126 (1999)

[11] A. Piskarskas, V. Smilgevicius, and A. Stabinis, "Optical parametric oscillation excited by an incoherent.conical beam", Opt. Comm. 143, 72-74 (1997)

[12] S. U. Alam, J. Nilsson, P. W. Turner, M. Ibsen, A. B. Grudinin, A. Chin, "Low cost multi-port reconfigurable erbium doped cladding pumped fiber amplifier", European Conference on Optical Communication 2000,

[13] G. P. Agrawal, Nonlinear Fiber Optics, 2 nd Edition, (Academic press, 1995), pp. 316-361 